Methods We retrospectively analyzed a consecutive series of 168 patients who presented with acute stroke symptoms and imaging revealing intracranial large vessel occlusion, who underwent cerebral angiography and attempted endovascular thrombectomy. Patients who underwent successful thrombectomy with no evidence of post-thrombectomy underlying ICAD lesion, dissection, or other vascular abnormality were presumed to have an embolic cause of their LVO. Patients who had an underlying vessel abnormality post-thrombectomy (exclusive of mechanical vasospasm) and no history of atrial fibrillation or other embolic risk factor were categorized as insituthrombosis.

Results Of the 168 patients, 151 arteries were revascularized with no post-thrombectomy vessel abnormality, indicating potential embolic cause. The remaining 17 patients had an underlying abnormality of which 15 appeared to be atherosclerotic in nature and 2 with underlying arterial dissection. These patients were initially indentified by the inability to pass the occlusive lesion with a J shape microwire. All 17 patients underwent angioplasty of their stenotic lesion at the time of thrombectomy. In addition, 13 patients underwent intracranial stenting. This included 8 patients stented acutely and 5 patients stented in a delayed fashion. Stenting was typically performed acutely if there was significant arterial recoil or lack of sufficient flow following angioplasty alone. One patient appeared to have an acute stent thrombosis within 12 hours of stenting.

Conclusions Although the majority of acute LVO stroke was embolic in this series, $10.1 \%$ of patients appeared to have a clinical picture more consistent with in-situthrombosis and $8.9 \%$ of the total patient cohort appeared to have an underlying ICAD lesion. Within this regional series, in-situ occlusion is a less common, but significant pathology, that may require different treatment strategies that conventional thrombectomy for LVO.

Disclosures M. Alexander: 2; C; Stryker Neurovascular, Medtronic, Penumbra, Inc. Z. Barnard: None.

\section{E-109 SHORT-TERM IN-HOSPITAL OUTCOMES OF THROMBOLYSIS FOR ACUTE ISCHEMIC STROKE PATIENTS WITH NON-PRIMARY BRAIN TUMORS AND ELECTROLYTE IMBALANCES}

K Tong*, A Wang-Selfridge, P Brauer. Anatomy, Kansas City University of Medicine and Biosciences, Kansas City, MO

\subsection{6/neurintsurg-2019-SNIS. 184}

Background Intravenous thrombolysis remains an underutilized treatment for acute ischemic stroke (AIS) due to several relative and absolute contraindications. Previous studies have found similar outcomes after thrombolysis between AIS patients with benign brain tumors and AIS patients without. This study aims to investigate short-term outcomes of thrombolytic treatment for the greater majority of AIS patients who have no history of primary brain tumors, particularly those with electrolyte imbalances.

Methods This retrospective cohort study utilized data from the 2012-2015Q3 Nationwide Inpatient Sample (NIS). ICD-9 codes identified adult patients (ages $18+$ ) who suffered acute ischemic stroke and received intravenous thrombolysis, and then further isolated patients diagnosed with electrolyte imbalances. Data for patients who were missing important clinical identifiers (age, gender, race, mortality), did not receive IV thrombolysis, and had primary brain tumors (benign or malignant) were excluded. Data analyses assessed hospital mortality rate, length of stay (LOS), inpatient charges, and average age of admission.

Results Of the 24692 encounters with AIS patients treated with thrombolysis and had no history of primary brain tumors, 5450were diagnosed with electrolyte imbalances (EI).

- Mean mortality rate of patients was significantly increased (10.4\% with EI vs. $6.0 \%$ with no EI, $\mathrm{p}<0.0001)$.

- Mean LOS was significantly longer (9.21 days with EI vs. 5.53 days with no EI, $\mathrm{p}<0.0001)$.

- Average total charges were significantly increased $(\$ 117,906.49$ with EI vs. $\$ 77,032.77$ with no EI, $\mathrm{p}<0.0001)$.

- Average age at admission was significantly older (67.88 years with EI vs. 66.81 years with no EI, p<0.0001)

Conclusion This study aims to inform physicians to better manage AIS patients receiving IV thrombolysis with electrolyte imbalances and no history of primary brain tumors. These patients experience higher mortality rate, longer LOS, increased total hospital charges, and older age at admission than those without EI. These findings suggest that placing clinical focus on managing electrolyte imbalances before administering IV thrombolysis for AIS may be critical for improving short-term in-hospital outcomes. Future research should aim to investigate different thrombolytic agents to determine the most optimal choice for patients with electrolyte imbalances.

Disclosures K. Tong: None. A. Wang-Selfridge: None. P. Brauer: None.

\section{E-110 ENDOVASCULAR TREATMENT DECISION IN ACUTE STROKE: DOES PHYSICIAN SEX MATTER? INSIGHTS FROM AN INTERNATIONAL MULTIDISCIPLINARY SURVEY}

${ }^{1}$ J Ospel, ${ }^{2} \mathrm{~N}$ Kashani, ${ }^{3} \mathrm{~B}$ Campbell, ${ }^{4} \mathrm{M}$ Foss, ${ }^{5} \mathrm{~F}$ Turjman, ${ }^{6} \mathrm{~S}$ Yoshimura, ${ }^{7} \mathrm{~A}$ Wilson, ${ }^{8} \mathrm{~W}$ Kunz, ${ }^{9} \mathrm{M}$ Cherian, ${ }^{10} \mathrm{~B}$ Kim, ${ }^{11} \mathrm{~A}$ Rabinstein, ${ }^{12} \mathrm{U}$ Fischer, ${ }^{13} \mathrm{P}$ Sylaja, ${ }^{14} \mathrm{~B}$ Baxter, ${ }^{15} \mathrm{~J} \mathrm{Heo}$, ${ }^{4} \mathrm{~B}$ Menon, ${ }^{16} \mathrm{G}$ Saposnik, ${ }^{4} \mathrm{M}$ Hill, ${ }^{17} \mathrm{M}$ Goyal ${ }^{*},{ }^{7} \mathrm{M}$ Almekhlafi. ${ }^{1}$ University Hospital Basel, Basel, Switzerland; ${ }^{2}$ Radiology, University of Calgary, Calgary, $A B$, Canada; ${ }^{3}$ Royal Melbourne Hospital, Melbourne, Australia; ${ }^{4}$ University of Calgary, Calgary, AB, Canada; ${ }^{5}$ Centre Hospitalier Universitaire de Lyon, Lyon, France; ${ }^{6}$ Neurosurgery, Hyogo College of Medicine, Hyogo, Japan; ${ }^{7}$ Radiology, Cumming School of Medicine, University of Calgary, Calgary, AB, Canada; ${ }^{8}$ Radiology, University Hospital, LMU Munich, Germany; ${ }^{9}$ Koval Medical center, Tamil Nadu, India; ${ }^{10}$ St. Mary's Hospital Seoul, Seoul, Korea, republic of ${ }^{11}$ Radiology, Mayo Clinic Rochester, Rochester, MN; ${ }^{12}$ University Hospital Bern, Bern, Switzerland; ${ }^{13}$ Sree Chitra Tirunal Institute for Medical Sciences and Technology, Thiruvanthapuram, India; ${ }^{14}$ Erlanger Medical Center, Chattanooga, $T N_{i}{ }^{15}$ Yonsei University, Seoul, Seoul, Korea, republic of; ${ }^{16}$ University of Toronto, Toronto, ON, Canada; ${ }^{17}$ Cumming School of Medicine, University of Calgary, Calgary, AB, Canada

\subsection{6/neurintsurg-2019-SNIS.185}

Introduction Numerous studies have compared treatment approaches of female and male physicians in different medical subspecialties, some of them revealed significant differences. To date, only few women are engaged in the neurointerventional field. Hence, it is unclear whether the treatment practice is influenced by physicians' sex. We explored whether there are differences in treatment decisions made by female and male physicians.

Materials and methods An international cross-sectional survey of both female and male stroke physicians and neurointerventionalists was conducted. Participants were randomly assigned 


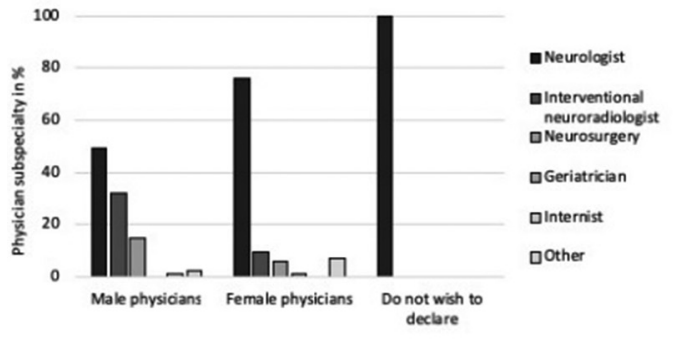

\section{Abstract E-110 Figure 1}

10 cases out of a pool of 22 case scenarios and asked how they would treat the patient A) assumed there were no economical or infrastructural constraints, and B) given their current working conditions. Subgroup analyses were performed for female and male physicians respectively.

Results 607 physicians (97 women, 508 men, 2 who did not disclose their sex), of different specialties (326 neurologists, 173 interventional neuroradiologists, 81 interventional neurosurgeons, 2 geriatricians, 5 internists, 20 other) from 38 countries participated in this survey. 6070 responses were obtained. Neurologists constituted the largest group of both female (76.3\%) and male (49.2\%) physicians, with a more even distribution of specialties in male physicians. Assuming ideal conditions, no significant differences in EVT decision making was observed (EVT was favored by $77 \%$ of female and $79.3 \%$ of male physicians). Under their current working conditions, female physicians decided less frequently in favor of EVT $(69.1 \%)$ as compared to their male colleagues $(76.9 \%$, $\mathrm{p}<0.001)$.

Conclusion Under the ideal conditions, EVT decision between male and female physicians did not differ. Current working conditions restricted female physicians' endovascular treatment decision to a greater degree as compared to their male colleagues, resulting in a significantly lower decision rate in favor of EVT.

Disclosures J. Ospel: None. N. Kashani: None. B. Campbell: None. M. Foss: None. F. Turjman: None. S. Yoshimura: None. A. Wilson: None. W. Kunz: None. M. Cherian: None. B. Kim: None. A. Rabinstein: None. U. Fischer: None. P. Sylaja: None. B. Baxter: None. J. Heo: None. B. Menon:None. G. Saposnik: None. M. Hill: None. M. Goyal: None. M. Almekhlafi: 1; C; unrestricted research grant by Stryker to University of Calgary.

\section{E-111 EFFICACY AND SAFETY OF ROBOTIC STEREOTACTIC ASSISTANCE (ROSA) DEVICE FOR MINIMALLY INVASIVE PLACEMENT OF INTRAPARENCHYMAL HEMATOMA AND INTRAVENTRICULAR CATHETERS: CASE SERIES}

N Alan*, V Kokkinos, A Faraji, N Agarwal, B Jankowitz. Neurosurgery, UPMC, Pittsburgh, $P A$

10.1136/neurintsurg-2019-SNIS. 186

Background In patients with supratentorial spontaneous intracerebral hemorrhage $(\mathrm{ICH})$, intrahematomal catheter placement may allow for clot thrombolysis and drainage. Robotic assistance may be used for the stereotactic placement of catheters. Objective To describe efficacy and safety of stereotactic robotic assistance for placement of intrahematomal catheters.

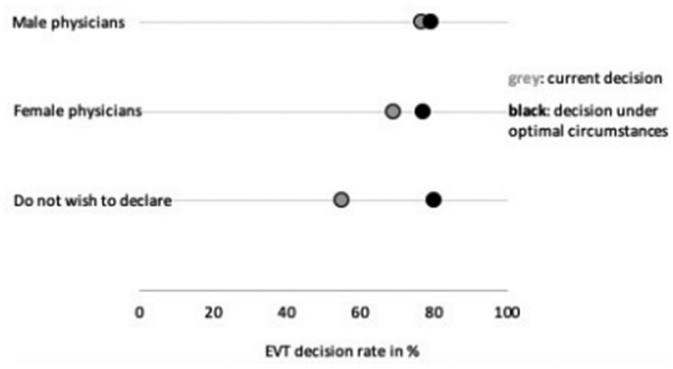

Methods From 2017-2018, 4 patients with average age 64 year old (three females, one male) who presented with supratentorial ICH underwent placement of intrahematomal catheter using stereotactic robotic assistance. Postoperatively, alteplase was delivered via the catheters to the clot and the hematoma drained.

Results A total of 9 catheters were placed. The time from last seen well to incision in the operating room was between 38 to 48 hours. Average volume of $\mathrm{ICH}$ at initial presentation was $52 \mathrm{cc}(29-68 \mathrm{cc})$ on volumetric analysis. Average dose of alteplase postoperatively was $6 \mathrm{mg}$ (range $5-9 \mathrm{mg}$ ), delivered over 32.5 hours (range 18-46 hours). Average volume of ICH at 12 hours after last dose of alteplase was 13 cc (range 2-34 cc) resulting in an average reduction of $39 \mathrm{cc}(77 \pm 24 \%)$. Output from the drains were 102-872 cc (average of $561 \mathrm{cc}$ ). Average error associated with catheter placement at its tip was $3.3 \mathrm{~mm} \pm 2.0 \mathrm{~mm}$, when comparing preoperative planned trajectory to immediate postoperative scan.

Conclusion Stereotactic robotic assistance was used safely and effectively for thrombolysis and drainage of supratentorial intracerebral hemorrhage.

Disclosures N. Alan: None. V. Kokkinos: None. A. Faraji: None. N. Agarwal: None. B. Jankowitz: None.

\section{E-112 HOW DO CLOTS RESPOND TO DIRECT ASPIRATION DURING INTERVENTIONAL TREATMENT OF ACUTE ISCHEMIC STROKE}

M Marosfoi, L Strittmatter, R Arslanian, C Raskett, M Gounis, A Puri, F Massari, K De Macedo Rodrigues, J Singh, J Chueh*. UMass Medical School, Worcester, MA

\subsection{6/neurintsurg-2019-SNIS.187}

Introduction Advances in pump design make aspiration patterns programmable, producing an infinite combination of cyclical aspiration profiles for clot removal during treatment of acute ischemic stroke (AIS). However, little is known about how clots respond to different aspiration patterns. The goal of this study is to assess the structural and mechanical changes of the clot under aspiration. It is our hypothesis that a decreased fibrin pore size correlates to a higher aspiration efficacy.

Materials and methods A fibrin-rich bovine clot was used to create an MCA occlusion in a patient-specific silicone model and underwent thromboaspiration via a $6 \mathrm{~F}$ Sofia Plus catheter (MicroVention, Tustin, CA). Three sets of the retrieved clots were prepared for scanning electron microscopy (SEM) analysis, including clots lodged at the MCA without aspiration (control) as well as clots subjected to static $(28 \mathrm{mmHg}$ ) or cyclic aspiration (amplitude: 18 to 27 